\title{
Microwave Photonic Filter: A Systematic Literature Review
}

\author{
Vijay Mishra ${ }^{1}$, Sumit Gupta ${ }^{2}$ \\ Student, Department of Electronics and communication, Oriental College of Technology, Bhopal, India ${ }^{1}$ \\ Associate Professor, Department of Electronics \& Communication, Oriental College of Technology, Bhopal, India ${ }^{2}$
}

\begin{abstract}
An overview of microwave photonic filters which are implemented in both incoherent and coherent operational regimes has been provided. For microwave photonic filters implemented in the incoherent regime, an incoherence light source usually a broadband light source such as a light-emitting diode source, an amplified spontaneous emission source, or a laser array is needed. Importance of negative coefficients and complex coefficients in photonic FIR filters is discussed in detail. In this paper various technique reported in the last few year for both incoherent and coherent regime microwave photonic filter are reviewed with significance on the system architecture of microwave photonic filters, photonic generation, processing of time-delay microwave photonic signals, tunable and reconfigurable microwave photonic filters. Complexity and challenges in system implementation for widespread use for practical application and possibilities of newer research in microwave photonic were also discussed.
\end{abstract}

Keywords: Photonic Filters, Microwave Photonics, Optical Source Generation, FIR, IIR, Laser Array.

\section{INTRODUCTION}

Microwave photonics as the name suggest is an interdisciplinary area where the interaction of microwave and optical signal is studied for various applications such as radar, sensor networks, broadband access network, filters, antennae array, fiber-to-home, satellite communication etc. Microwave photonics started to emerge in late 80s when it was felt by the researchers that to transport microwave signals in electrical domain is quite difficult because of the reasons such as: Bulkiness of the devices, heating effects, high losses at higher frequencies, low bandwidth and almost zero tunablity of high frequency filters. To counter these losses microwave photonics came in picture possessing advantages that can never be achieved in electrical domain, advantages such as light weight, almost no heating effect, high bandwidth, high tune-ability of optical filters, almost no electromagnetic interference etc. Basically, Microwave photonics covers wide variety of topics such as photonic generation of microwave and mm-wave signal, photonic microwave signal processing, optically controlled phase array system, radio-over-fiber system and photonic analog to digital conversion.

In this paper we will discuss about photonic processing of microwave signals, more specifically, about microwave photonic filters. Wilner and Van den Heuvel were one of the first researchers who proposed that optical fibers could be used as delay lines to make a photonic filter. After this revolutionary concept microwave photonic filters came into picture. In the past few years tremendous amount of efforts have been made to implement different kind of microwave photonic filter. The most common application of microwave photonic filters is in the field of warfare applications, radar, optical communication, satellite communication, optical networks. This article will also review both Incoherent and Coherent photonic filters in detail.

\section{LITERATURE SURVEY}

A. Types of microwave photonic filters:

a) Photonic filters of Incoherent regime

To setup a microwave filter in optical domain in a topic of interest for many researchers and recently many setups of photonic filters have been proposed in recent years. Incoherent photonic filters are first of its kind. They are implemented using optical fibers as delay lines and the gain at each optical fiber determines the coefficient of the filter. This delay line configuration closely implements FIR or IIR filters in optical regime. The multiple taps are realized using either a sliced broadband light source or a laser array, with the time delay between two adjacent taps achieved by passing a microwave modulated optical signal through optical paths having different physical lengths or traveling in a single fiber or waveguide with linearly chromatic dispersion [1].

1. Delay line filters with positive coefficients:

Photonic delay-line microwave filter with an FIR consists of a light source; a modulator, generally implemented using Mach-Zehnder modulator (MZM) or a phase modulator (PM); a delay-line module; and a photodetector (PD). The key 
device in a microwave photonic filter is the optical delay-line module, which can be implemented using an array of fiber Bragg gratings (FBGs), an arrayed waveguide, a chirped FBG (CFBG), or a dispersive fiber. For N Tap delay line FIR configuration is given by:

$$
\mathrm{h}(\mathrm{t})=\sum_{\mathrm{k}=0}^{\mathrm{N}-1} \alpha_{\mathrm{k}} \delta(\mathrm{t}-\mathrm{KT})
$$

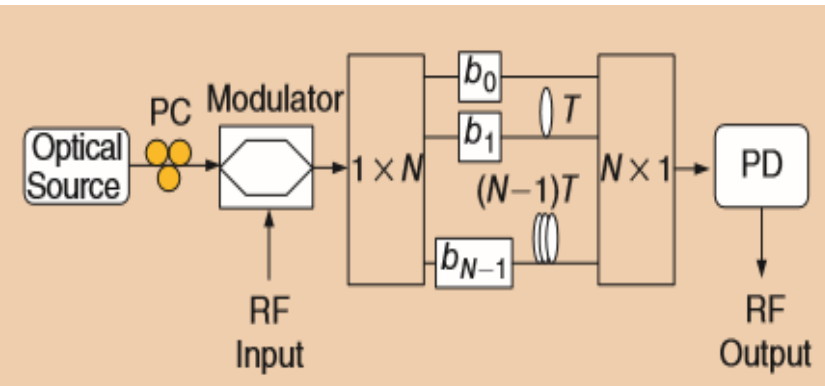

Fig1. Delay-line microwave photonic filter with an FIR [1].PC: Polarization controller

Delay-line microwave photonic filter has been introduces to avoid optical interference in the incoherent regime. Two different source using broadband light source and laser array where observed in microwave photonic filter configuration in incoherent regime. Broadband light source and RF signals combined through modulator and sent to array of FBGs where signals sliced by FBGs in array and reflected through optical circulator, where as in laser array multiple wavelengths multiplex with addition to RF signals through modulator and sent through dispersive fiber to the photodetector.

Once, the time delay line if fabricated in FBGs tuning will be difficult. so tuning can be performed by tuning wavelength spacing . The tuning speed in laser array is in microsecond, faster tuning speed in the range of nanosecond can be achieve using optical comb, demonstration at speed of 40ns was reported [3]. It is observed that delay line microwave photonic filter operating incoherent regime with all positive coefficients operates as a low pass filter. To overcome this limitation, microwave photonic filter with negative or complex coefficient arbitrary filter is designed in the incoherent regime.

\section{2) Delay line filters with negative coefficients:}

To implement a band pass FIR filter negative coefficients are necessary. To implement negative coefficients differential detection is most common method. There are other techniques to produce negative coefficients as well such as using cross gain modulation, differential MZM, using PM-IM conversion techniques etc.

One approach among the several techniques proposed for negative coefficient is to use wavelength conversion based on cross-gain modulation (XGM) in semiconductor optical amplifier (SOA) [5]. A tunable laser source with wavelength $\chi_{1}$ modulated by RF signals splits into two parts, one goes through optical fibre with time delay other combines with continuous wave laser source of different wavelength $\lambda_{2}$ and goes through SOA. With effects of XGM in SOA there is phase inversion of $\pi$ in the CW beam $\lambda_{2}$ is also modulated by RF signal led to the generation of negative coefficient. An optical bandpass filter is used for filtering out the residual signal at $\lambda_{1}$. Now the time-delay signal in upper channel and phase inverted signal in lower channel combines and detected at PD. Microwave photonic filter with negative coefficient is realized using bandpass filter. Detection at PD is incoherent because generated wavelength is from two different laser sources.

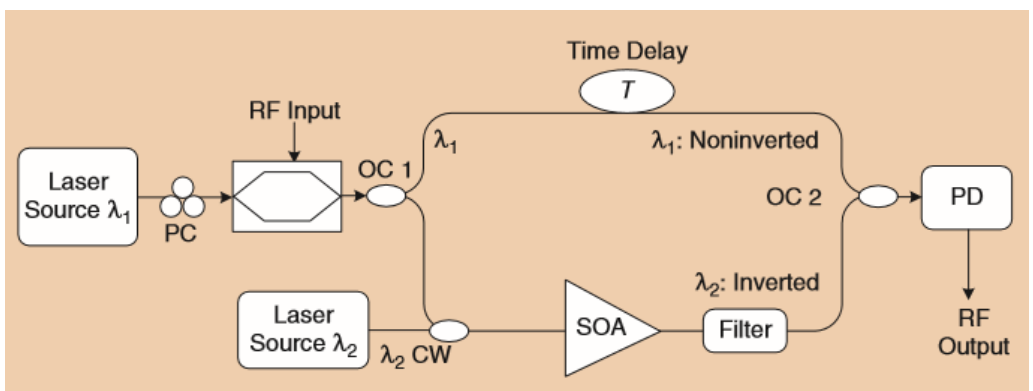

Fig2 A delay-line microwave photonic filter with a negative coefficient based on XGM in an SOA [5].

A technique to generate negative coefficient is based on two MZMs biased at complimentary transmission slopes was reported [6]. At the output of PD two complementary microwave signals is generated with respect to positive and 
negative coefficients. To generate microwave delay-line with multi taps positive and negative coefficient, a multiwavelength laser array is required to different wavelength to the two MZMs. Using single MZMs wavelength depends on transfer function of MZMs, was reported [7]. A dc bias make MZM operated at complementary slope when optical wavelengths are at $1310 \mathrm{~nm}$ and $1550 \mathrm{~nm}$ windows.

\section{3) Delay line filters with complex coefficients:}

With the change in time delay difference for frequency tuning in photonics filter led to change in bandwidth and spectral response. While requirement of many system is only change in center frequency of passband or stopband but the shape of frequency spectral response remains same. A delay-line complex coefficient microwave photonic filter is designed to overcome this problem.

Transfer function with complex coefficient $\mathrm{N}$-tap microwave delay-line filter given by

$$
\begin{aligned}
& \mathrm{H}(\omega)=\mathrm{a}_{0}+\mathrm{a}_{1} \mathrm{e}^{-\mathrm{j} \theta} \cdot \mathrm{e}^{-\mathrm{j} \omega \mathrm{T}}+\cdots+\mathrm{a}_{\mathrm{N}-1} \mathrm{e}^{-\mathrm{j}(\mathrm{N}-1) \theta} \cdot \mathrm{e}^{-\mathrm{j} \omega(\mathrm{N}-1) \mathrm{T}} \\
& \quad=\sum_{\mathrm{n}=0}^{\mathrm{N}=1} \mathrm{a}_{\mathrm{n}} \mathrm{e}^{-\mathrm{j} \omega \theta} \cdot \mathrm{e}^{-\mathrm{j} \omega \mathrm{n} \mathrm{T}}
\end{aligned}
$$

Where, $\mathrm{T}$ is the time delay difference. In (2) phase shift of all taps were in fixed relationship for same FSR. Hence, the phase shift of each tap should be tuned independently. Microwave photonic delay-line filter with complex coefficient was reported [8]. In [8], a two tap photonic microwave delay-line with one complex coefficient was implemented using system consists of three optical attenuator and two microwave couplers.

Transfer function of the filter is given by:

$$
\mathrm{H}(\mathrm{f})=\cos (2 \pi \mathrm{fT}+\varphi)
$$

Where $t$ is constant, by tuning the phase $\varphi, H(f)$ will be shifted along horizontal direction without changing its shape .Equation can be expressed as

$$
\begin{gathered}
H(f)=\frac{a}{2}\left(e^{j \omega T}+e^{-j \omega T}\right)-\frac{b}{2 j}\left(e^{j \omega T}-e^{-j \omega T}\right. \\
=e^{-j \omega T}\left\{\frac{a}{2}\left(e^{j \omega 2 T}+1\right)-\frac{b}{2 j}\left(e^{j \omega 2 T}-1\right)\right\}
\end{gathered}
$$

Where $\mathrm{a}=\cos (\varphi), \mathrm{b}=\sin (\varphi)$. It is observed that filter has one complex coefficient $-\frac{\mathrm{b}}{2 \mathrm{j}}$. As seen from equation (4) In [9] microwave photonic filter with tunable delay-line with complex coefficient was reported, where complex coefficient was generated by changing in phase of RF signals, realization were done through combined effect if single sideband (SSB)modulation and stimulated Brillouin scattering (SBS). Phase of microwave signal carried through optical carrier will results phase shifts if spectrums fall SSB gain spectrum passing through optical fiber [10].In [9] to trigger SSB high -power Erbium-doped fiber amplifier is used for generation of complex coefficient. System is complicated due to MZM and long fibre consumes considerable powers at high cost. A simplified configuration system is reported in [11] using wideband-tunable optical RF phase shifter consists of two MZM where phase shift is simply done by adjusting the bias voltage.

\section{Photonic filters with non-uniformly spaced delay-line configuration:}

Non-uniformly speed taps for complex coefficient by introducing additional delay to taps [12]. Impulse response $\mathrm{h}_{R}(\mathrm{~T})$ given by

$$
\mathrm{h}_{\mathrm{R}}(\mathrm{T})=\sum_{\mathrm{k}=0}^{\mathrm{N}-1} \mathrm{a}_{\mathrm{k}} \delta(\mathrm{t}-\mathrm{kT})
$$

Where, $N$ is the no of taps, $a_{k}$ is filter coefficient of kth taps, $T=2 \pi / \Omega$.

By applying Fourier transform to (5), frequency response of microwave photonic filter is given by

$$
\mathrm{H}_{\mathrm{R}(\omega)=\sum_{\mathrm{k}=0}^{\mathrm{N}-1} \exp \left[\left(i-j \mathrm{j} \frac{2 \pi}{\Omega} \omega\right)\right.}
$$

Phase term introduced into specific coefficient by adding an additional time delay know as time-delay based on phase shift [12] As the tunablity of a filters increases it is essential that the bandwidth of the filter remains unchanged thus the complex coefficient filters came into picture, the phase component of coefficients helps the filter to maintain the bandwidth of filter even of the filter is tuned to other frequency as well. The filter with complex coefficient in frequency domain can expressed as:

$$
H^{\prime}(\omega)=\sum_{k=0}^{N-1} \alpha_{k} \exp \left[-j\left(k \frac{2 \pi}{\Omega}+\Delta \tau_{k}\right) \omega\right]
$$




$$
\begin{aligned}
& =\sum_{\mathrm{k}=0}^{\mathrm{N}-1} \alpha_{\mathrm{k}} \exp \left(-\mathrm{j} \omega \Delta \tau_{\mathrm{k}}\right) \cdot \exp \left(-\mathrm{jk} \frac{2 \pi}{\Omega} \omega\right) \\
& =\sum_{\mathrm{k}=0}^{\mathrm{N}-1} \alpha_{\mathrm{k}} \exp \left(-\mathrm{jm} \cdot \Omega \Delta \tau_{\mathrm{k}}\right) \cdot \exp \left(-\mathrm{jk} \frac{2 \pi}{\Omega} \omega\right)
\end{aligned}
$$

Where, $\alpha_{\mathrm{k}} \exp \left(-\mathrm{jm} . \Omega \Delta \tau_{\mathrm{k}}\right)$ is the complex coefficient of the filter.

In uniformly spaced microwave photonic delay-line filter, frequency response is different for different channel. The technique to design a non-uniformly spaced microwave delay-line filter is particularly useful for applications such as RF pulse phase encoding [15], arbitrary microwave waveform generation [16], and pulse compression [17].

\section{b) Photonic filters of coherent regime:}

Till now the filters discussed above are of incoherent regime which uses delay line concept to make an IIR or FIR filters, also, to reduce the optical interference broadband light source or laser array source. However, coherent filters are newly emerged filters which does not use delay line to make a filters, instead they use a single light source. A basic coherent microwave photonic filter consist of narrow-line width light wave from a laser source is sent to a phase modulator. Assuming small signal modulation, at the output of the PM, an optical carrier with two sidebands is generated. It should be noted that for phase modulation, the two sidebands produced are out of phase. Thus, to detect a phase-modulated signal directly at a PD produces a dc voltage since the beating between the optical carrier and the lower sideband will completely counters the beating between the optical carrier and the upper sideband. However, if upper and or lower band is removed by an optical notch filter in transmission or by filters, such as an FBG or two cascaded FBGs [18], then the phase-modulated signal is converted into an SSB intensity-modulated signal, and SSB intensity-modulated signal at a PD will generate RF signal as shown in figure(3). One of the major limitations of the technique [18] is large passband as uniform FBG was used for the filtering purpose. Using ring resonator [19], the passband can be smaller, but it is too large for many application.

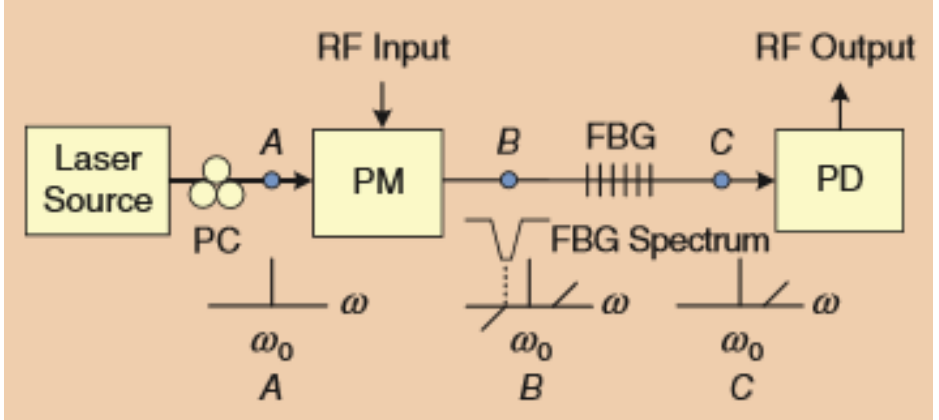

Fig(3). A coherent microwave photonic filter, in which an optical notch filter is used to filter out one sideband of a phase-modulated signal, thus achieving PM-IM conversion [18]

Some other techniques are also introduced in this regime such as by implementing PM-IM conversion using optical notch filters, and then using optical heterodyning to gain get the RF signal, another setup is being proposed in which two fiber Bragg grating are used to filter out the central frequency and a side band of the signal which provided more compact bandwidth. Due to ultra-narrow bandwidth of nonlinear properties of an optical fiber such as SBS, its application is implemented in one of the setup which helped to gain ultra-narrow bandwidth photonic filter. The setup mainly consist an EDFA (Erbium doped fiber amplifier), highly nonlinear SMF (single mode fiber), Dual parallel Mach-Zehnder modulator.

Advantage of this system is that the filter can be tune by tuning local oscillator frequency, and very narrow passband. Due to high-power EDFA and long SMF, system is complicated. To generate unbalanced double sided band modulation system in configure by replacing PM with another DP-MZM [20]. If the power ratio of two side bands is controlled continuously, then microwave notch filter turn into tunable notch depth or can be controlled to become bandpass filter with tunable passband gain [21].To overcome the limitation of [20] one may use an optical filter with ultra-narrow passband, using PS-FBG for PM-IM conversion [22].

Other coherent filters also consist of dual band filters and flat top filters.

a) Coherent Microwave photonic filters with a flat Top.

The concept of PM-IM converter is again used to translate spectral response used to implement photonic filter with flattop [24]. Designed super structured FBG (SFBG) is used instead of SSB or PS-FBG. SFBF is modulated by periodic 
sampling function. A $\pi$ phase shift is introduced to grating, leading to narrow notch; technique is known an EPS technique [25]. The reflection bandwidth and notch width of the SFBG, determines the tunable range and bandwidth of the passband, can be controlled by regulating the length and maximum index modulation of the SFBG. Figure 4(a) shows a simulated reflection spectrum of a PS-FBG with a single phase shift, and Figure 4(b) shows a simulated reflection spectrum of an SFBG with two phase shifts. Figure 4(c) shows the structure of an SFBG in which two EPSs are incorporated.

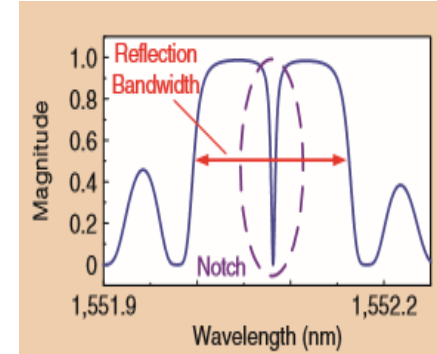

(a)

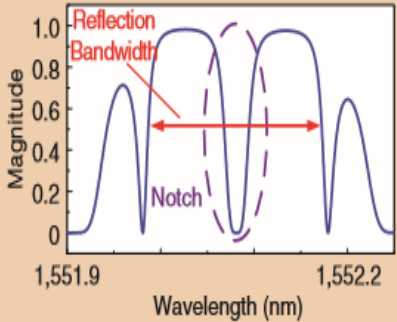

(b)

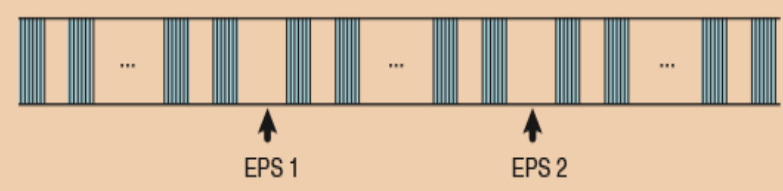

(c)

Fig4. (a) Simulated reflection spectrum of a PS-FBG with a single phase shift. (b) Simulated reflection spectrum of an SFBG with two phase shifts. (c) The SFBG structure [24]

\section{b) Coherent Microwave photonic filters with dual Passbands}

Due to increase in demand for multiband/multifunctional microwave photonic system, microwave filter with dual passbands are needed for various modern services. Same concept has been discussed earlier to implement a microwave photonic filter with two passband [26]. Configuration is similar to EPS technique [25].The EPS-FBG has multi-channel due to spatial sampling of garting structure. EPSs introduced to \pm 1 channel are realized with change in sampling function. In the design, two $\pi$ phase shift are introduced to both of the \pm 1 channel to produce an ultranarrow and flattop notch in each channel. Simulated reflection spectrum of an EPS-FBG with two $\pi$ phase shift shown in fig 6 .

To produce two independent, optical carriers from TLSs (TLS1 and TLS2) tuned to locate at channel \pm 1 of EPS-FBG, as shown in fig 5. As two $\pi$ phase shift introduces to obtain flat-bottom notch, the passband have small shape factor defines between ratio of 20 and $3 \mathrm{~dB}$ bandwidth. Passband of central frequency of $\omega_{1}$ and $\omega_{2}$ will produce due to PMIM conversion at sideband of phase modulated signal suppressed by notch in channel \pm 1 channel. The central frequencies of passband can be tuned independently by shifting the wavelength of TLS1 and TLS2.

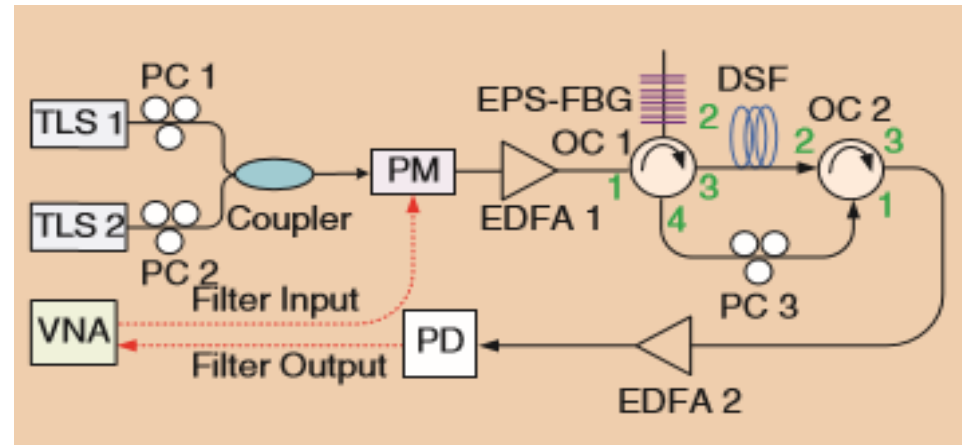

Fig5. A schematic of a dual-passband microwave photonic filter [26]. VNA: vector network analyser.

Spectral response of dual passband filters with bandwidth of $167.3 \mathrm{MHz}$ and shape factor of 3.8 for first passband and bandwidth of $143.4 \mathrm{MHz}$ and shape factor of 3.3 for second passband, as shown in fig 6. The first and second passband have Frequencies tunable range of 5.4 and $7.4 \mathrm{GHz}$, respectively. Variations of magnitude over tunable range are maintained within $\pm 0.5 \mathrm{~dB}$. [26]. Performance of the filter are defined by two important parameter that is SFDRs for two passband and noise figure(NFs) that can be improved by partially suppressing the optical carrier by using SSBassisted filter shown in fig 22 . This is equivalent to increasing the filter gain. 


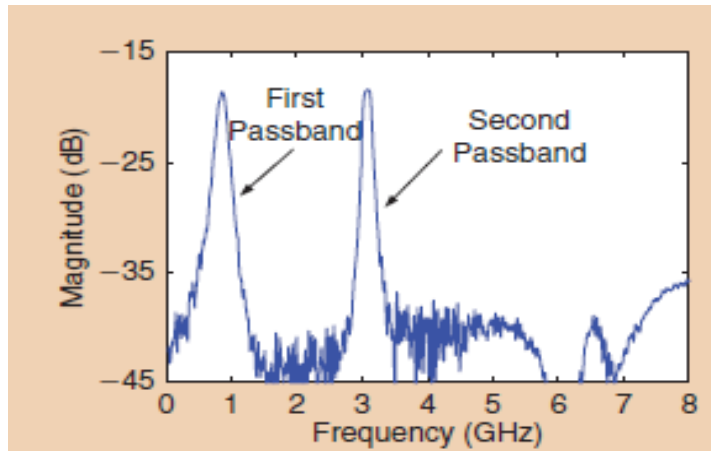

(a)

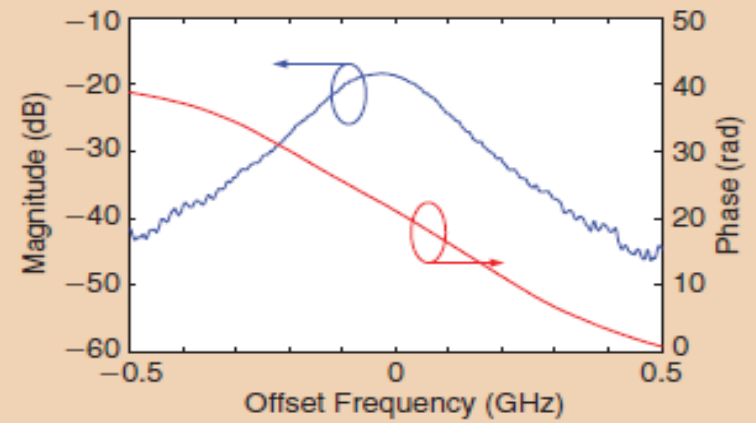

(b)

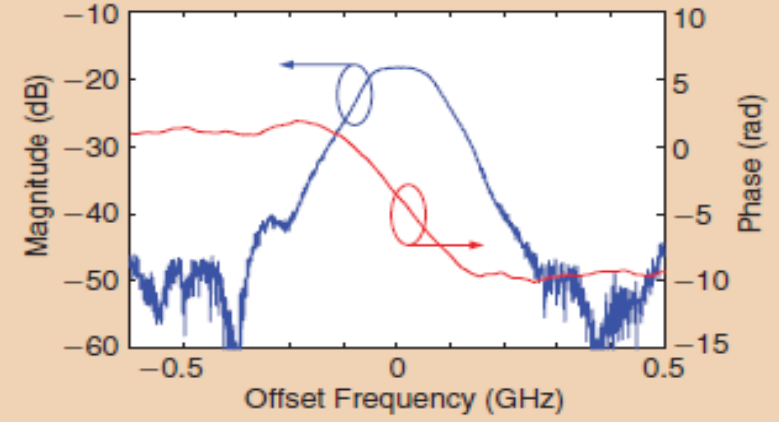

(c)

Fig6. (a) The frequency response of the dual-passband microwave photonic filter. (b) The frequency response and phase response of the first passband. (c) The frequency response and phase response of the second passband [26]

\section{III.CONCLUSION}

For microwave photonic filters implemented in the incoherent regime, an incoherence light source usually a broadband light source such as a light-emitting diode source, an amplified spontaneous emission source, or a laser array is needed. On the other hand, the implementation of microwave photonic filters in the coherent regime can have a much simpler configuration. The filter spectral response is, in fact, the spectral response of an optical filter that is translated into the microwave domain. Thus, the key to implementing a coherent microwave photonic filter is to design an optical filter with a well-defined spectral response. Tuning coherent microwave photonic filter can be simply accomplished by tuning the wavelength of the coherent light source. At last, to make laser sources using bandgap engineering could be the ultimate efforts to reduce the size of filters. One possible solution is to employ hybrid integration, to produce light emission and light amplification using a direct band gap material and using silicon for other functions (modulation, time delay, and photo-detection). With this hybrid approach [27], fully integrated microwave photonic filters would perform better and be ready for practical applications.

\section{REFERENCES}

[1] J. P. Yao, “Microwave photonics,” J. Light wave Technol., vol. 27, no. 3, pp. 314-335, Feb. 2009.

[2] J. Sancho, J. Bourderionnet, J. Lloret, S. Combrié, I. Gasulla, S. Xavier, S. Sales, P. Colman, G. Lehoucq, D. Dolfi, J. Capmany, and A. de Rossi, "Integrable microwave filter based on a photonic crystal delay line,” Natl. Commun., vol. 3, article 1075, pp. 1-9, Sept. 2012. 
[3] V. R. Supradeepa, C. M. Long, R. Wu, F. Ferdous, E. Hamidi, D. E. Leaird, and A. M. Weiner, "Comb-based radiofrequency photonic filters with rapid tunability and high selectivity," Natl. Photon., vol. 6, no. 5, pp. 186-194, May 2012.

[4] S. Sales, J. Capmany, J. Marti, and D. Pastor, "Experimental demonstration of fiber-optic delay line filters with negative coefficients," Electron. Lett., vol. 31, no. 13, pp. 1095-1096, June 1995.

[5] F. Coppinger, S. Yegnanarayanan, P. D. Trinh, and B. Jalali, "Alloptical RF filter using amplitude inversion in a semiconduct or optical amplifier,” IEEE Trans. Microwave Theory Tech., vol. 45, no. 8, pp. 1473-1477, Aug. 1997

[6] J. Capmany, D. Pastor, A. Martinez, B. Ortega, and S. Sales, "Microwave photonics filter with negative coefficients based on phase inversion in an electro-optic modulator," Opt. Lett., vol. 28, no. 16, pp. 1415-1417, Aug. 2003

[7] B. Vidal, J. L. Corral, and J. Marti, “All-optical WDM multi-tap microwave filter with flat bandpass,” Opt. Exp., vol. 14, no. 2, pp. 581-586, Jan. 2006

[8] N. You and R. A. Minasian, “A novel tunable microwave optical notch filter,” IEEE Trans. Microw. Theory Tech., vol. 49, no. 10, pt. 2, pp. 2002-2005, Oct. 2001

[9] A. Loayssa, J. Capmany, M. Sagues, and J. Mora, "Demonstration of incoherent microwave photonic filters with all-optical complex coefficients," IEEE Photon. Technol. Lett., vol. 18, no. 16, pp. 1744-1746, Aug. 2006.

[10] A. Loayssa, R. Hernandez, D. Benito, and S. Galech, "Characterization of stimulated Brillouin scattering spectra by use of optical singlesideband modulation," Opt. Lett., vol. 29, no. 6, pp. 638-640, Mar. 2004.

[11] Y. Yan and J. P. Yao, "A tunable photonic microwave filter with a complex coefficient using an optical RF phase shifter," IEEE Photon. Technol. Lett., vol. 19, no. 19, pp. 1472-1474, Sept. 2007

[12] Y. Dai and J. P. Yao, "Nonuniformly-spaced photonic microwave delay-line filter," Opt. Expr., vol. 16, no. 7, pp. 4713-4718, Mar. 2008.

[13] F. Zeng and J. P. Yao, “All-optical bandpass microwave filter based on an electro-optic phase modulator,” Opt. Exp., vol. 12, no. 16, pp. 38143819, Aug. 2004.

[14] F. Zeng and J. P. Yao, "Investigation of phase modulator based all-optical bandpass microwave filter," J. Lightwave Technol., vol. 23, no. 4, pp. 1721-1728, Apr. 2005.

[15] Y. Dai and J. P. Yao, "Microwave pulse phase encoding using a photonic microwave delay-line filter," Opt. Lett., vol. 32, no. 24, pp. 34863488, Dec. 2007.

[16] Y. Dai and J. P. Yao, "Chirped microwave pulse generation using a photonic microwave delay-line filter with a quadratic phase response," IEEE Photon. Technol. Lett., vol. 21, no. 9, pp. 569-571, May 2009.

[17] Y. Dai and J. P. Yao, "Microwave correlator based on a nonuniformly spaced photonic microwave delay-line filter," IEEE Photon. Technol. Lett., vol. 21, no. 14, pp. 969-971, July 2009

[18] X. Yi and R. A. Minasian, “Microwave photonic filter with single bandpass response,” Electron. Lett., vol. 45, no. 7, pp. 362-363, Mar. 2009.

[19] J. Palací, G. E. Villanueva, J. V. Galán, J. Martí, and B. Vidal, "Single bandpass photonic microwave filter based on a notch ring resonator," IEEE Photon. Technol. Lett., vol. 22, no. 17, pp. 1276-1278, Sept. 2010.

[20] D. Marpaung, R. Pant, B. Morrison, and B. J. Eggleton, "Frequency agile microwave photonic notch filter with anomalously-high stopband rejection," Opt. Lett., vol. 38, vol. 21, pp. 4300-4303, Nov. 2013.

[21] X. Han and J. P. Yao, "Bandstop-to-bandpass microwave photonic filter using a phase-shifted fiber Bragg grating," submitted for publication.

[22] W. Li, M. Li, and J. P. Yao, "A narrow-passband and frequencytunable microwave photonic filter based on phase-modulation to intensitymodulation conversion using a phase-shifted fiber Bragg grating,” IEEE Trans. Microwave Theory Tech., vol. 60, no. 5, pp. 1287-1296, May 2012.

[23] T. Erdogan, "Fiber grating spectra," J. Lightwave Technol., vol. 15, no. 8, pp. 277-1294, Aug. 1997.

[24] L. Gao, X. Chen, and J. P. Yao, "Microwave photonic filter with a narrow and flat-top passband," IEEE Microwave Wireless Compon. Lett., vol. 23, no. 7, pp. 362-364, July 2013.

[25] Y. Dai, X. Chen, D. Jiang, S. Xie, and C. Fan, "Equivalent phase shift in a fiber Bragg grating achieved by changing the sampling period," IEEE Photon. Technol. Lett., vol. 16, no. 10, pp. 2284-2286, Oct. 2004.

[26] L. Gao, J. Zhang, X. Chen, and J. P. Yao, "Microwave photonic filter with two independently tunable passbands using a phase modulator and an equivalent phase-shifted fiber Bragg grating," IEEE Trans. Microwave Theory Tech., vol. 62, no. 2, pp. 380-387, Feb. 2014.

[27] M. J. R. Heck, J. F. Bauters, M. L. Davenport, J. K. Doylend, S. Jain, G. Kurczveil, S. Srinivasan, Y. Tang, and J. E. Bowers, "Hybrid silicon photonic integrated circuit technology," IEEE J. Select. Topics Quantum Electron., vol. 19, no. 4, article 6100117, July-Aug. 2013. 